\title{
HLA-DRB1* alleles and symptoms associated with Heerfordt's syndrome in sarcoidosis
}

\author{
P. Darlington*,\# , L. Tallstedt ${ }^{\star}$, L. Padyukov ${ }^{+}$, I. Kockum ${ }^{\S}$, K. Cederlund ${ }^{f}$, \\ A. Eklund*,\# and J. Grunewald*,*
}

ABSTRACT: Heerfordt's syndrome (HS) consists in its complete form of uveitis, parotid or salivary gland enlargement and cranial nerve palsy. The objective of the present study was to analyse if there are also links between HLA-DRB1* alleles and HS, as it is a specific phenotype of sarcoidosis.

1,000 patients with sarcoidosis, out of whom 83 had symptoms associated with HS, were included in the study together with a group of 2,000 healthy individuals from the same population, matched for sex and age. HLA-DRB1* allelic groups were determined for all individuals, and comparisons were made between different disease subgroups and between patients and healthy controls.

We found that the HLA-DRB1*04 allele was overrepresented in patients with symptoms associated with HS. $83(8.3 \%)$ of all patients had one or more of the symptoms and $46(55 \%)$ of them were HLA-DRB1*04 positive. 44 (55\%) of the patients with ocular sarcoidosis, i.e. the most common symptom associated with HS, were HLA-DRB1*04 positive, compared with $35.9 \%$ of healthy controls $(p=0.0008)$, and only $26.6 \%$ of the whole group of sarcoidosis patients $(p<0.0001)$.

HLA-DRB1*04 seems to protect against overall sarcoidosis but appears to be a significant risk factor for ocular sarcoidosis as well as for other manifestations associated with HS.

KEYWORDS: Eyes, Heerfordt's syndrome, human leukocyte antigen, ocular sarcoidosis, sarcoidosis

arcoidosis is a systemic granulomatous disease of unknown aetiology. It is characterised by formation of noncaseating granulomas in the affected organs. The lungs and/or thoracic lymph nodes are engaged in $>90 \%$ of all cases but almost any organ, such as the eyes, skin, heart and the nervous system can be involved [1,2]. The course of the disease varies significantly. Many patients recover but some develop chronic disease with pulmonary fibrosis and eventually respiratory failure. The first line of treatment is oral corticosteroids. Treatment is usually initiated in patients with progressive loss of lung function, but extrapulmonary manifestations, such as involvement of the heart, eyes and nervous system, are also common indications for treatment [1].

Earlier studies have shown that there are links between certain HLA class II alleles and distinct clinical manifestations of sarcoidosis, such as the well-known association of HLA-DRB $1^{*} 03$ and Löfgren's syndrome (LS) [3, 4]. LS is characterised by an acute onset usually with fever, bilateral ankle arthritis and/or erythema nodosum and bilateral hilar lymphadenopathy with, in some cases, parenchymal infiltrates [5]. LS is common in Scandinavia but very rare in patients of Japanese origin in whom DRB1*0301 is virtually missing [6]. Heerfordt's syndrome (HS) is another, more unusual manifestation of sarcoidosis described in the literature. This syndrome was originally termed "Febris uvea-parotidea subchronica" and was described in 1909 by Heerfordt [7]. He described three patients with uveitis, parotid swelling, cranial nerve palsy and fever. In 1937, WALDENSTRÖM [8] classified it as a distinct manifestation of sarcoidosis. The complete form of HS is considered to consist of uveitis, parotid or salivary gland enlargement and cranial palsy, especially facial, and fever is also common [9].

In patients with ocular sarcoidosis, uveitis is by far the most common manifestation and sometimes co-exists with symptoms associated with HS, such as cranial nerve palsy and engagement of parotid and/or salivary glands. The incidence of eye involvement in patients with sarcoidosis varies from $5 \%$ in Finland to $>70 \%$ in Japan, supporting the notion of a genetic influence on

\section{AFFILIATIONS}

*Dept of Medicine, Division of Respiratory Medicine,

'Dept of Ophtalmology, St. Erik Eye Hospital,

${ }^{+}$Dept of Medicine Solna, Rheumathology Unit,

${ }^{\S}$ Dept of Clinical Neurosciences, ${ }^{f}$ Dept of Clinical Science, Intervention and Technology (CLINTEC), Karolinska Institutet, and \#Division of Respiratory Medicine, Karolinska University Hospital, Stockholm, Sweden.

CORRESPONDENCE $P$. Darlington

Dept of Medicine, Division of Respiratory Medicine

Södersjukhuset (Stockholm South General Hospital)

Sjukhusbacken 10 11883 Stockholm Sweden

E-mail: pernilla.darlington@ sodersjukhuset.se

Received:

Feb 092011

Accepted after revision: April 182011 First published online: May 122011 
signs of disease presentation [10-12]. Other examples of eye involvement are lacrimal gland inflammation, optic neuritis and conjunctival granulomas. Commonly, both eyes are engaged [11]. Anterior uveitis is reported to be more common in AfricanAmericans (70-75\%) while posterior uveitis is more common in Caucasians (65-83\%) [13]. In patients with sarcoidosis with anterior uveitis, treatment with topical corticosteroids is commonly sufficient, while posterior uveitis usually requires more frequent monitoring and is more difficult to treat [12].

Since HS is a distinct phenotype of sarcoidosis, and there is a strong genetic influence as suggested by sharp differences in disease phenotypes in patients with different ethnic origin, we hypothesised that there might be associations between specific HLA-DRB1* alleles and HS. The objective of the present study was therefore to analyse whether there are links between HLADRB1* alleles and symptoms associated with HS, such as uveitis, engagement of parotid and/or salivary glands or cranial nerve palsy, with particular focus on uveitis.

\section{MATERIALS AND METHODS}

\section{Study subjects}

A total of 1,000 HLA-typed patients were included in the study, of whom 383 were classified as having LS. All those included were consecutive patients referred to the outpatient clinic at the Division of Respiratory Medicine, Karolinska University Hospital, Solna, Sweden, but patients from the South General Hospital, Stockholm, Sweden, were also included. All patients were referred for diagnostic investigation and activity assessment to the respective outpatient clinic. The vast majority of patients were seen by one of the authors (A. Eklund), from 1994 onwards. Patients were diagnosed with sarcoidosis through typical clinical and radiographic manifestations, findings at bronchoscopy with bronchoalveolar lavage (BAL), including an elevated CD4/CD8 cell ratio, and positive biopsies, using the criteria outlined by the World Association of Sarcoidosis and other Granulomatous disorders (WASOG) [14]. All patients with ocular involvement were examined by ophthalmologists and had findings compatible with ocular sarcoidosis according to International Criteria for the Diagnosis of Ocular Sarcoidosis [11]. In total, 80 out of the 1,000 patients were found to have ocular sarcoidosis i.e. inflammatory activity located in the eye, secondary to the sarcoidosis disease. Only patients with symptoms originating in the eyes (such as pain, photophobia and blurred vision) were seen by an ophthalmologist, in the vast majority by one of the authors (L. Tallstedt) at the St. Erik Eye Hospital, Stockholm, Sweden. Patients with uveitis were classified according to anterior, posterior, unilateral or bilateral involvement. Ocular manifestation with only iridocyclitis without signs of posterior uveitis were defined as anterior uveitis and patients with vitreous floaters/snowballs, venous vasculitis, neovascularisation, choroidal granulomas, macular oedema, papilloedema or chororetinal peripheral lesions were classified as having posterior uveitis. Patients were judged to have involvement of the parotid and salivary glands if this was biopsy-proven and/or if there was an obvious enlargement and simultaneously co-existing findings were compatible with sarcoidosis. Patients were defined as having neurosarcoidosis according to Zajicek criteria (in this study, only patients with cranial nerve palsy and co-existing findings compatible with sarcoidosis were included) [15]. Chest radiographs were evaluated and findings staged by one of the authors ( $\mathrm{K}$. Cederlund), who is subspecialised in chest radiology, with a long experience of interstitial pulmonary disease. Chest radiographs were controlled at the time of diagnosis and at $2 \mathrm{yrs}^{\prime}$ follow-up. Chest radiographs in patients with sarcoidosis were classified into five stages: stage 0 , normal; stage I, bilateral hilar lymphadenopathy; stage II, bilateral lymphadenopathy with parenchymal infiltrates; stage III, parenchymal infiltrates alone; and stage IV, fibrotic bands and volume retraction [16]. The study was approved by the regional ethical committee and all patients included gave their informed consent.

All 1,000 patients were characterised by age, sex, HLA-type, treatment/no treatment, resolving/nonresolving disease and LS/non-LS (table 1). Nonresolving disease was defined as disease duration $>2$ yrs with remaining signs of active disease as evaluated by the chest radiographic findings, recurrent uveitis and other disease parameters (i.e. fatigue, cough and fever). Patients were in general followed at the outpatient clinic for $\geqslant 2$ yrs and longer if signs of active disease. Symptoms associated with HS were defined as uveitis, engagement of parotid and/or salivary glands and cranial nerve palsy. Patients with uveitis and one of the other symptoms were regarded having an incomplete form of HS and patients with all three symptoms to have the complete form. The control group consisted of healthy individuals from the same population, 1,138 females and 862 males, and were matched for the distribution of age and sex with the patients.

\begin{tabular}{|c|c|c|c|}
\hline \multirow[t]{2}{*}{ TABLE 1} & \multicolumn{3}{|c|}{$\begin{array}{l}\text { Clinical characteristics in patients with ocular } \\
\text { sarcoidosis, all sarcoidosis patients and healthy } \\
\text { controls }\end{array}$} \\
\hline & $\begin{array}{c}\text { Ocular } \\
\text { sarcoidosis }\end{array}$ & $\begin{array}{c}\text { All patients } \\
\text { with sarcoidosis }\end{array}$ & $\begin{array}{l}\text { Healthy } \\
\text { controls }\end{array}$ \\
\hline Subjects $\mathrm{n}$ & 80 & 1000 & 2000 \\
\hline \multicolumn{4}{|l|}{ Sex } \\
\hline Males & 40 & 570 & 1138 \\
\hline Females & 40 & 430 & 862 \\
\hline Age yrs & 37 (19-75) & 38 (9-78) & $42(15-73)$ \\
\hline \multicolumn{4}{|c|}{ Radiographic stage } \\
\hline 0 & 13 & 61 & \\
\hline 1 & 40 & 484 & \\
\hline$\|$ & 21 & 323 & \\
\hline III & 6 & 104 & \\
\hline IV & 0 & 28 & \\
\hline Never-smoker & 38 & 553 & \\
\hline Ever-smoker & 40 & 433 & \\
\hline Unknown & 2 & 14 & \\
\hline Löfgren & $10^{\#}$ & 383 & \\
\hline Non-Löfgren & $70^{\#}$ & 617 & \\
\hline Resolving & $6^{\#}$ & 409 & \\
\hline Nonresolving & $64^{\#}$ & 493 & \\
\hline Unknown & 10 & 98 & \\
\hline $\begin{array}{l}\text { Data are prese } \\
\text { patients without } \\
\text { the Materials an }\end{array}$ & $\begin{array}{l}\text { ocular sarcoidosis. For } \mathrm{d} \\
\text { d Methods section. }\end{array}$ & $\begin{array}{l}\text { nge). }{ }^{\#}: p<0.0001 \\
\text { finition of the radiogra }\end{array}$ & $\begin{array}{l}\text { mpared with } \\
\text { hic stage, see }\end{array}$ \\
\hline
\end{tabular}




\section{HLA typing}

Genomic DNA was extracted from whole blood samples and HLA-DRB1* allelic groups were determined (table 2) for every patient using the PCR-sequence-specific primers (SSP) technique (Olerup SSP DR Low Resolution Kit; Olerup SSP AB, Saltsjöbaden, Sweden) [17]. For the controls, the majority were HLA-typed using PCR-SSP but some of the HLA-DRB1* alleles were determined with restriction length polymorphism $[18,19]$.

\section{Statistical analysis}

Data were analysed by the Chi-squared test or, in the case of small numbers, by Fisher's exact test. Statistical analyses were performed with Graph Pad Prism 4.03 (Graphpad Software Inc., San Diego, CA, USA). When comparing different allele frequencies, $\mathrm{p}<0.003$ was regarded significant (after Bonferroni correction for the number of alleles $(n=13)$, i.e. dividing 0.05 with $13=0.003)$. Otherwise $\mathrm{p}<0.05$ was regarded significant.

\section{RESULTS}

Among all 1,000 HLA-typed sarcoidosis patients, 80 (8\%) were found to have eye involvement and, out of these $80,72(90 \%)$ had uveitis, $19(2 \%)$ were found to have engagement of parotid or salivary glands and facial nerve palsy occurred in $11(1 \%)$ (fig. 1). Uveitis, involvement of parotid or salivary glands and cranial nerve palsy are three different symptoms known to be associated with HS, and simultaneous presence of all symptoms represents the complete form of this syndrome. The majority of patients with ocular engagement had uveitis, often bilateral and usually a prolonged disease course. In the patients with involvement of parotid and salivary glands, they were bilateral in eight cases and unilateral in nine cases (unknown in two cases). In all cases, the salivary gland enlargement gradually subsided. The cranial nerve palsy was unilateral and transient in all 11 cases. In patients with symptoms associated with HS, the diagnosis was verified with biopsy in the majority of patients and most of them were taken

\section{TABLE 2 Carrier frequency of HLA-DRB1 alleles}

\begin{tabular}{|c|c|c|c|}
\hline & $\begin{array}{l}\text { Control } \\
\text { subjects }\end{array}$ & $\begin{array}{l}\text { All patients with } \\
\text { sarcoidosis }\end{array}$ & $\begin{array}{c}\text { Ocular } \\
\text { sarcoidosis }\end{array}$ \\
\hline Subjects $\mathrm{n}$ & 2000 & 1000 & 80 \\
\hline *01 & 22.3 & $12.7^{\#}$ & $7.5^{\circ}$ \\
\hline *03 & 22.7 & $37.9^{\#}$ & 18.8 \\
\hline *04 & 35.9 & $26.6^{\#}$ & $55.0^{\circ}$ \\
\hline *07 & 15.9 & $11.4^{\circ}$ & 10.0 \\
\hline *08 & 8.4 & 8.9 & 10.0 \\
\hline *09 & 3.4 & 2.5 & 1.2 \\
\hline *10 & 1.1 & 1.7 & 1.2 \\
\hline *11 & 12.5 & 12.4 & 5.0 \\
\hline *12 & 4.2 & 5.2 & 5.0 \\
\hline *13 & 26.6 & 26.3 & 20.0 \\
\hline *14 & 4.1 & $8.5^{\#}$ & 7.5 \\
\hline *15 & 27.6 & $33.1^{\bullet}$ & 36.3 \\
\hline *16 & 1.5 & 0.7 & 1.2 \\
\hline
\end{tabular}

Data are presented as $\%$, unless otherwise stated. ${ }^{\#}: p<0.0001$ compared with healthy controls; $"$ : p-value $<0.003$ compared with healthy controls. from parotid and salivary glands, peripheral lymph nodes, skin lesions, lungs and, in rare cases, also from the eyes. The remaining patients had, with the exception of the extrapulmonary manifestations, typical chest radiograph findings, LS, high CD4/CD8 ratio in BAL fluid and/or a elevated serum ACE level. 13 patients had radiological stage 0 and eight of these patients had the diagnose confirmed via biopsy taken from peripheral lymph nodes, skin biopsies or parotid glands. The other five were patients that met the criteria; they had typical ocular findings as judged by an experienced ophthalmologist and laboratory tests supporting the diagnosis.

Out of all patients $(n=1,000), 83(8.3 \%)$ had uveitis and/or engagement of parotid or salivary glands and/or cranial palsy and $46(55 \%)$ of them were HLA-DRB1*04 positive. $40(55 \%)$ of the patients with uveitis were HLA-DRB1*04 positive, 10 (53\%) of the patients with involvement of parotid and salivary glands and $8(73 \%)$ of the patients with cranial nerve palsy also carried the same allele. Out of the 83 patients with at least one of the symptoms associated with HS, there were $16(19 \%)$ patients who had two or three of the symptoms, 10 (62.5\%) were HLADRB1*04 positive (figs $2 \mathrm{a}$ and b). Among the 80 patients with ocular sarcoidosis, eight patients had no uveitis and were therefore not counted as having symptoms associated with HS. Several of these patients had conjuctival granulomas. These eight patients were included, however, in the whole group of patients with ocular sarcoidosis (i.e. inflammatory activity located in the eye, secondary to the sarcoidosis disease). Four of these patients $(50 \%)$ were HLA-DRB1*04 positive.

HLA-DRB1*04 was present in 266 (26.6\%) of all 1,000 patients compared with $718(35.9 \%)$ in healthy controls $(p<0.0001)$. The HLA-DRB ${ }^{*} 04$ allele was strongly connected to the HLA-DQB1*03 allele (data not shown). Among the 266 HLADRB1*04-positive patients, $46(17 \%)$ had uveitis and/or engagement of parotid and salivary glands and/or cranial nerve palsy. Among the 734 HLA-DRB1*04-negative patients, these combinations occurred in only $37(5 \%)$ patients $(p<0.0001)$. Thus,

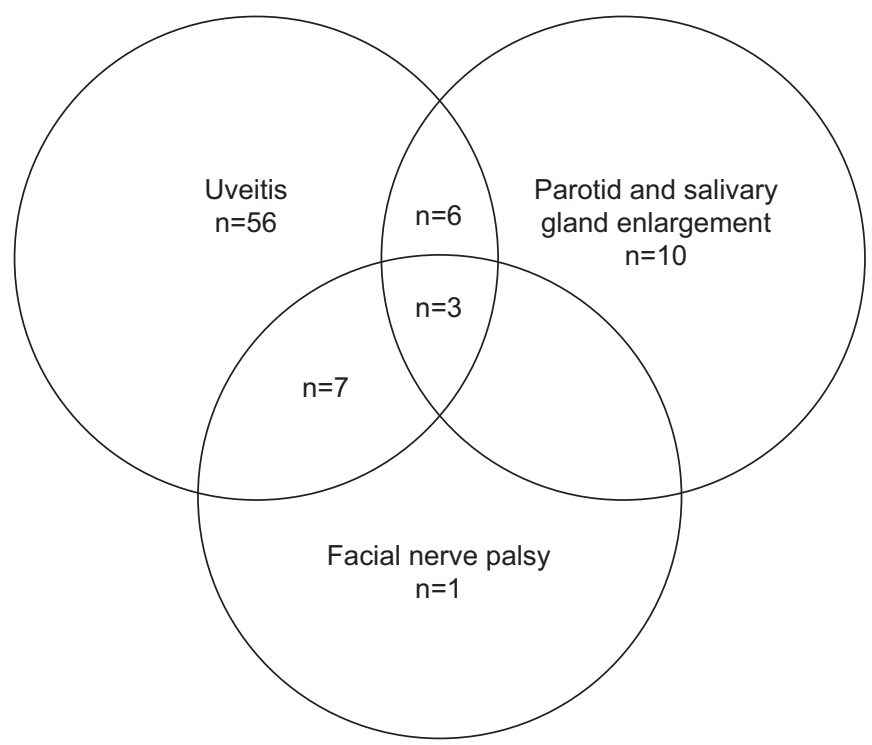

FIGURE 1. Patients with uveitis, parotid and salivary gland enlargement and facial nerve palsy. As shown, some of the patients had two or three symptoms. 

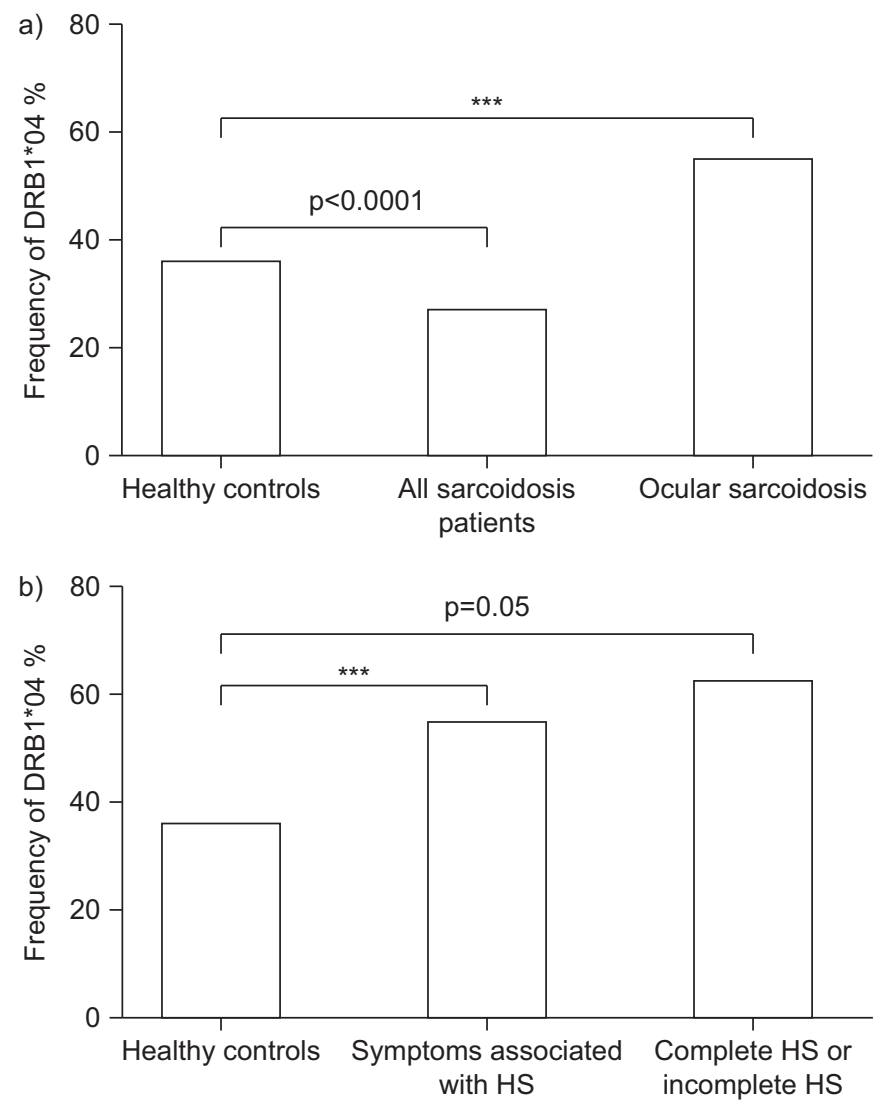

FIGURE 2. a) Frequency of HLA DRB $1 * 04$ in healthy controls $(n=2,000)$, all sarcoidosis patients $(n=1,000)$ and patients with ocular sarcoidosis $(n=80)$. b) Frequency of HLA DRB ${ }^{*} 04$ in healthy controls $(n=2,000)$, in patients with symptoms associated with Heerfordt's syndrome (HS; uveitis, parotid and/or salivary gland enlargement and cranial nerve palsy)( $(n=83)$, and in patients with incomplete HS $(n=16)$, i.e. simultaneous occurrence of at least two of the three symptoms or all three of them (complete HS). p-values show a comparison with healthy controls. ${ }^{* \star}: \mathrm{p}<0.001$.

there was a 3.4 times increased risk for having HS-associated manifestations in HLA-DRB1*04-positive patients. $21(2.1 \%)$ of the whole group of sarcoidosis patients were homozygous for HLA-DRB ${ }^{*} 04$ and seven (33\%) of them had eye disease; in two cases this was concomitant with facial palsy. Patients homozygous for HLA-DRB1*04 therefore had a 6.6 times increased risk for HS-associated symptoms (fig. 3). 33 (75\%) out of the 44 HLA-DRB1*04-positive patients with ocular sarcoidosis were subtyped, and $28(85 \%)$ of them carried the HLA-DRB1*0401 allele (fig. 4).

In patients with ocular sarcoidosis, other extrapulmonary manifestations were common, as 32 patients $(40 \%)$ had at least one other manifestation (e.g. skin lesions, cranial nerve palsy and hypercalcaemia).

LS was less common in patients with ocular sarcoidosis $(\mathrm{p}<0.0001)$ compared with patients without. The HLADRB1*03 allele significantly associated with this syndrome was also less common among patients with eye involvement compared with patients without $(\mathrm{p}=0.0002)$. The HLA-DRB $1 * 03$ allele was also less common among patients with the incomplete

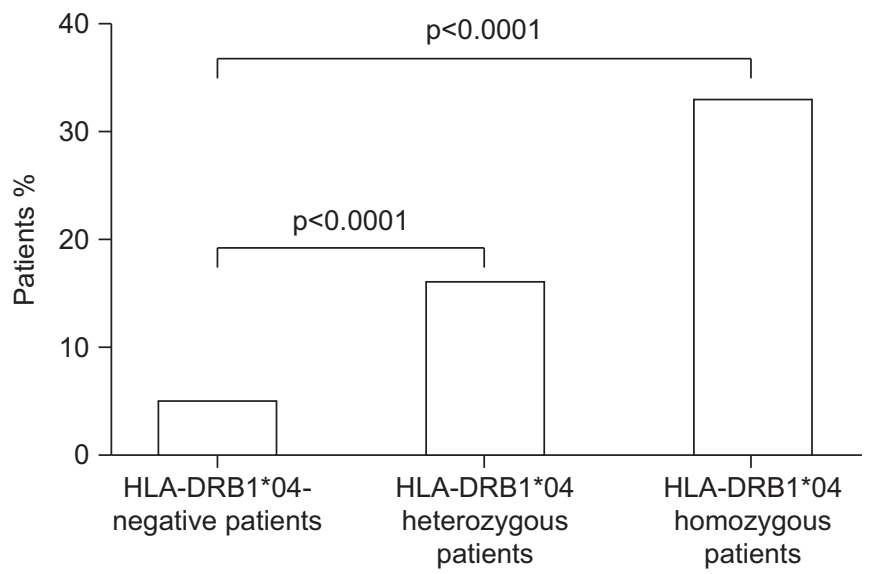

FIGURE 3. Frequency of patients with symptoms associated with Heerfordt's syndrome (uveitis, parotid and/or salivary gland enlargement and cranial nerve palsy) among HLA-DRB1*04 negative $(n=734), H L A-D R B 1 * 04$ heterozygous $(n=245)$ and HLA-DRB $1{ }^{*} 04$ homozygous patients $(n=21)$. The $p$-value of the frequency is in comparison with HLA-DRB1*04-negative patients.

or complete form of HS, where only two (12.5\%) had the allele. In addition, the frequency of HLA-DRB1*01 was significantly lower among patients ocular sarcoidosis compared with healthy controls, and also seemed to be protective against contracting overall sarcoidosis (table 2).

Most of the patients with ocular engagement had uveitis $(n=72)$. Among 68 patients for whom we had access to more detailed information, $36(53 \%)$ had posterior uveitis, and the uveitis was bilateral in $45(66 \%)$ (table 3$)$. The localisation of uveitis did not differ significantly between the HLA-DRB1*04positive and -negative patients.

58 of the patients with ocular sarcoidosis were followed by chest radiograph at onset of disease and 2 yrs later. After 2 yrs, chest radiograph showed enlarged lymph nodes and/or infiltrates in $49(84.5 \%)$ out of 58 patients (fig. 5). In 70 patients with ocular engagement, where there was information available regarding disease course, only six had a resolving disease and four of these had LS. As shown in figure 6a, there was no obvious difference between HLA-DRB1*04-negative and HLA-DRB1*04-positive

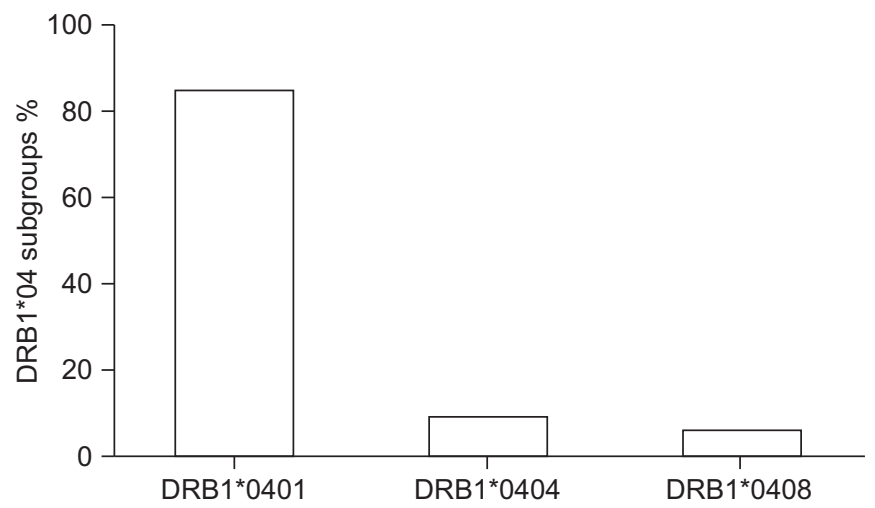

FIGURE 4. HLA subtyping of DRB1*04-positive patients with ocular sarcoidosis $(n=33)$. Results are presented as the percentage of patients belonging to each subgroup. DRB1*0401: $n=28$; DRB1*0404: $n=3$; DRB1*0408: $n=2$. 


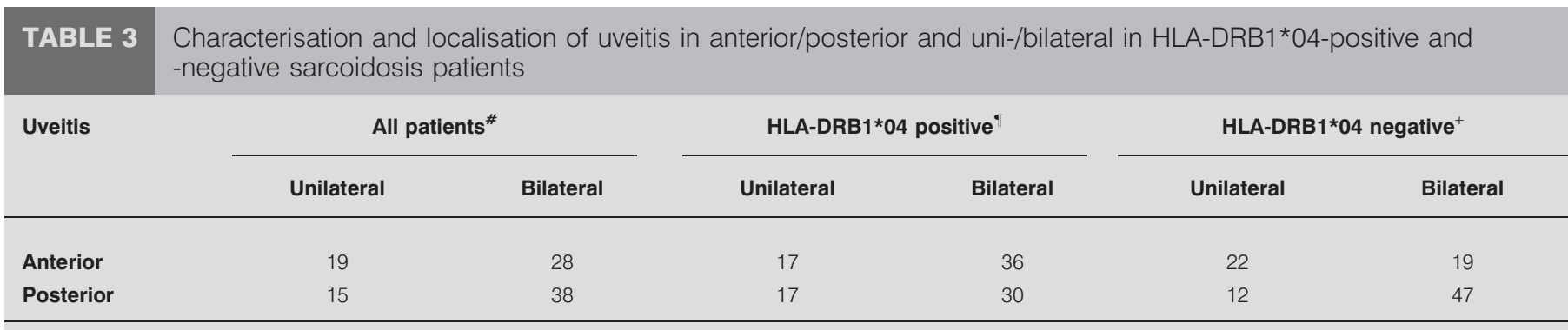

Data are presented as \%. Note that out of 72 patients with ocular symptoms compatible with Heerfordt's syndrome (i.e. uveitis), there was information about 68 as to whether it was posterior or anterior. ${ }^{\#}: n=68 ; n=36 ;{ }^{+}: n=32$.

patients with ocular sarcoidosis with regard to disease progression. Among HLA-DRB1*04-positive patients without ocular engagement $(n=202), 101(50 \%)$ had a resolving disease (fig. $6 b)$ compared with only five $(12.5 \%)$ with ocular disease $(\mathrm{p}<0.0001)$.

\section{DISCUSSION}

Complete HS consists of bilateral uveitis, parotid or salivary gland swelling and cranial nerve palsy. The complete form of the syndrome is rare, as also described by SCADDING [9]. Among our 1,000 patients, only three showed the complete syndrome. In accordance with Scadding's modification of HS, we divided the patients into incomplete or complete forms of HS. We also added patients with only uveitis and patients without uveitis but with the other two manifestations, and described them as having symptoms associated with HS. Fever is described as common in HS but we chose to exclude this parameter, as information about the body temperature was usually lacking. Christian Heerfordt, who originally described the syndrome, did not include pulmonary engagement as a part of the syndrome [7]. In our study, 67 (81\%) of the patients with HS-associated symptoms had thoracic involvement. The patients with cranial nerve palsy and parotid or salivary glands were few. It is, however, interesting that 10 out of 11 patients with cranial nerve palsy also had uveitis and this strong connection strengthens the indication that this is a syndrome. Half of the patients with parotid and salivary gland enlargement did not have ocular engagement and six out of 10

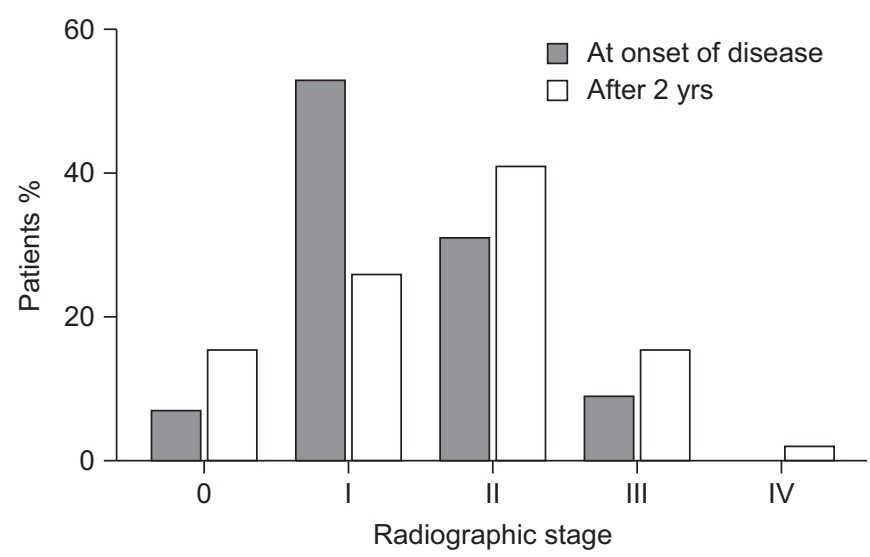

FIGURE 5. 58 patients with ocular sarcoidosis and their radiographic stage at onset of disease and 2 yrs after diagnosis. Results are presented as percentage of patients who belong to radiographic stages $0-I V$, respectively. were HLA-DRB1*04 positive. This also indicates that the connection to HLA-DRB1*04 is not only driven by the uveitis. The high frequency of HLA-DRB ${ }^{*} 04$ in these patients seems to be a possible genetic link, which could explain why these symptoms known to be part of HS occur simultaneously. Out of the patients with ocular inflammatory manifestations other than uveitis, half of them were HLA-DRB1*04 positive.

We found an overrepresentation of HLA-DRB1*04, particularly HLA-DRB1*0401, in patients with the HS-associated manifestations uveitis, parotid and salivary glands, as well as cranial nerve palsy. In this as well as in some previous studies, HLADRB1*04 was otherwise shown to be protective against sarcoidosis [20,21]. There are other studies that support our findings of a genetic association to symptoms associated with HS. For example, associations between HLA-DRB1*0401 and

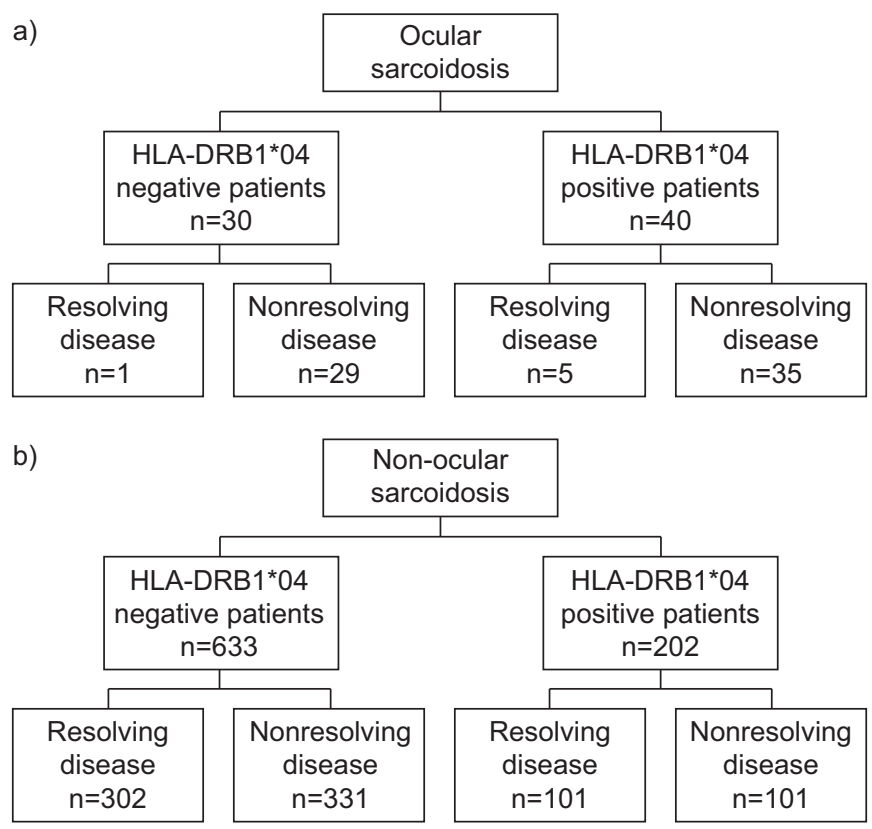

FIGURE 6. a) 70 patients with ocular sarcoidosis, where there was information available regarding resolving or nonresolving disease. The patients are divided into HLA-DRB $1{ }^{*} 04$ positive and negative, respectively. The numbers of patients with resolving and nonresolving disease are given. b) Corresponding data on outcome of the disease in $202 \mathrm{HLA}-\mathrm{DRB} 1{ }^{*} 04$ positive and $633 \mathrm{HLA}-\mathrm{DRB} 1{ }^{*} 04$ negative patients without ocular involvement. There was a significant difference in outcome depending on whether they had ocular or nonocular sarcoidosis $(p<0.0001)$. 
ocular sarcoidosis have been suggested, but in significantly smaller patient cohorts and where patients of different ethnic origins were included [22, 23]. Correlations between HLADRB1*0401 and involvement of parotid and salivary glands were noted in a study by ROSSMAN et al. [22]; however, the correlation was significant only for African-Americans and not for Caucasians. In a more recent study by SATO et al. [23], a link between DRB1*0803 and neurosarcoidosis was reported in Japanese patients as well as between DRB1*0401/DQB1*0301 and uveitis in a UK population. Previous studies have also shown genetic associations for uveitis in sarcoidosis with the HSP70/Hom rs2075800 G allele in patients from the UK and with the CTLA-4 gene polymorphism in patients from Japan [24, 25]. There are also studies about genetic similarities between inflammatory bowel disease and sarcoidosis, both of which are associated with an increased risk for uveitis [26, 27]. One possible explanation of the genetic linkage with a specific HLAallele is cross-reactivity, for example, there are proteins in the eye that resemble the antigen that the immune system initially reacted against. According to this theory, HLA-DRB1*04 allows an adequate antigen presentation of eye-derived proteins. Patients who are homozygous for HLA-DRB1*04 may have a more efficient antigen presentation, leading to an inflammatory reaction.

In our study, the uveitis was often bilateral and posterior and no significant differences were found between the HLADRB1*04-positive and -negative patients. The presence of the HLA-DRB $1 * 03$ allele, previously shown to be associated with a good prognosis in Scandinavian patients [4, 28], was significantly reduced in patients with ocular sarcoidosis. However, in all patients, the frequency of the allele was higher compared with healthy controls and it seemed to be associated with an increased risk of contracting sarcoidosis. HLA-DRB1*01, which was previously found to strongly protect against non-LS [21], was also found to protect against eye involvement (table 2).

$80(8.0 \%)$ patients were found to have ocular sarcoidosis and this prevalence is low compared with several other studies, probably for several reasons. One explanation may be our relatively strict criteria for the patients diagnosed with ocular sarcoidosis. Only patients with obvious symptoms were examined for eye involvement. For example, patients with conjunctivitis sicca were excluded in our study but included in others $[29,30] .70$ patients were followed for 2 yrs, and among these we found no significant difference in outcome between the HLA-DRB1*04-positive or -negative patients. In comparison with patients without ocular sarcoidosis, patients with eye involvement had a significantly poorer prognosis with higher frequency of nonresolving disease. Therefore, ocular engagement seems to be of greater prognostic importance than HLA type. This is in contrast to patients with LS, in whom the HLA type (HLA-DRB1*03) could be a prognostic marker [2, 4]. Ethnicity is also likely to have an impact on the prevalence of ocular sarcoidosis; for example, it is known that in Japan the incidence is considerably higher than in Scandinavia [10]. Most of the patients with ocular sarcoidosis in our study had nonresolving disease, which sharply differs from the Japanese sarcoidosis patients in whom ocular engagement is often associated with a good prognosis [31]. In a study by PiEtinalho et al. [32], where Finnish and Japanese patients with sarcoidosis were compared, ocular sarcoidosis was found in more than half of the Japanese patients compared with $5 \%$ in the Finnish cohort. The majority of the Japanese patients had a normal chest radiograph after 2 yrs [32].

The vast majority of the patients were from one single centre, and likely to be representative of the general population as these patients are normally investigated at a respiratory department rather than in an outpatient clinic. However, a possible bias against mild forms of sarcoidosis could not be ruled out. Patients with one episode of uveitis might not always be investigated for sarcoidosis and patients with only cranial nerve palsy are probably not routinely investigated with chest radiograph.

In conclusion, we set out to analyse associations between HLADRB1* alleles and symptoms linked to HS in a large wellcharacterised, homogenous Scandinavian sarcoidosis population, and we specifically investigated ocular involvement. The results of our study show that there is a significant correlation between HLA-DRB ${ }^{*} 04$ and symptoms linked to HS. However, in sharp contrast to LS, where a genetic association is also known, HS has a prolonged disease course. Therefore, it seems reasonable to suggest that HLA-DRB1*04-positive patients should be closely monitored for particularly uveitis, but also parotid or salivary gland enlargement and cranial nerve palsy. Involvement of one of these organs also calls for increased awareness of possible nonresolving disease and a more intensive follow-up.

\section{SUPPORT STATEMENT}

This study was supported by The Swedish Heart-Lung Foundation, The King Oscar II Jubilee Foundation, the Swedish Research Council, Torsten and Ragnar Söderberg's Foundation, and Karolinska Institutet. Support was also given through the regional agreement on medical training and clinical research (ALF) between Stockholm County Council and the Karolinska Institutet. None of the funding sources had any influence on the production of this manuscript.

\section{STATEMENT OF INTEREST}

None declared.

\section{ACKNOWLEDGEMENTS}

The authors would like to thank H. Blomquist, B. Engvall, B. Dahlberg, M. Dahl, G. De Forest, A. Roos (all from the Dept of Medicine Solna, Respiratory Medicine Unit, Karolinska Institutet and the Division of Respiratory Medicine, Karolinska University Hospital, Stockholm, Sweden) and B. Hedquist (Eye Clinic, Södersjukhuset, Stockholm, Sweden) for their assistance. The Swedish Childhood diabetes study, Diabetes Incidence Study in Sweden and Epidemiological Investigation of Multiple Sclerosis (EIMS) and EIRA study group are acknowledged for sharing HLA genotypes from their controls.

\section{REFERENCES}

1 Newman LS, Rose CS, Maier LA. Medical progress - sarcoidosis. N Eng J Med 1997; 336: 1224-1234.

2 Iannuzzi MC, Rybicki BA, Teirstein AS. Sarcoidosis. N Eng J Med 2007; 357: 2153-2165.

3 Hedfors E, Lindstrom F. HLA-B8/Dr3 in sarcoidosis - correlation to acute onset disease with arthritis. Tissue Antigens 1983; 22: 200-203.

4 Berlin M, Fogdell-Hahn A, Olerup O, et al. HLA-DR predicts the prognosis in Scandinavian patients with pulmonary sarcoidosis. Am J Respir Crit Care Med 1997; 156: 1601-1605.

5 Löfgren S. Erythema nodosum: studies on etiology and pathogenesis in 185 adult cases. Acta Med Scand 1946; 124: Suppl. 174, 1-197. 
6 Grunewald J, Shigematsu M, Nagai S, et al. T-cell receptor V gene expression in HLA-typed Japanese patients with pulmonary sarcoidosis. Am J Respir Crit Care Med 1995; 151: 151-156.

7 Heerfordt CF. [Über eine "Febris uveo-parotidea subchronica" an der Glandula parotis und der Uvea des Auges lokalisiert und häufug mit Paresen cerebrospinaler Nerven kompliziert.] Arch Ophthalmol 1909; 70: 254-273.

8 Waldenström J. Some observations on uveoparotitis and allied conditions with special reference to the symptoms from the nervous system. Acta Med Scand 1937; 91: 53-68.

9 Scadding JG. Sarcoidosis. London, Eyre and Spottiswoode, 1967; pp. 200-202.

10 Pietinalho A, Ohmichi M, Hiraga Y, et al. The prognosis of pulmonary sarcoidosis in Finland and Hokkaido, Japan - a comparative study. Sarcoidosis 1992; 9: 443-444.

11 Herbort CP, Rao NA, Mochizuki M. International criteria for the diagnosis of ocular sarcoidosis: results of the First International Workshop on Ocular Sarcoidosis (IWOS). Ocul Immunol Inflamm 2009; 17: 160-169.

12 Baughman RP, Lower EE, Kaufman AH. Ocular sarcoidosis. Semin Respir Crit Care Med 2010; 31: 452-462.

13 Rothova A. Ocular involvement in sarcoidosis. Brit J Ophthalmol 2000; 84: 110-116.

14 ATS/ERS/WASOG. Statement on sarcoidosis. Joint Statement of the American Thoracic Society (ATS), the European Respiratory Society (ERS) and the World Association of Sarcoidosis and Other Granulomatous Disorders (WASOG) adopted by the ATS Board of Directors and by the ERS Executive Committee, February 1999. Am J Respir Crit Care Med 1999; 160: 736-755.

15 Zajicek JP, Scolding NJ, Foster O, et al. Central nervous system sarcoidosis diagnosis - management. QJM 1999; 92: 103-117.

16 Scadding JG. Prognosis of intrathoracic sarcoidosis in England - a review of 136 cases after 5 years observation. BMJ 1961; 2: 1165-1172.

17 Olerup O, Aldener A, Fogdell A. HLA-DQB1 and HLA-DQA1 typing by PCR amplification with sequence-specific primers (PCRSSP) in 2 hours. Tissue Antigens 1993; 41: 119-134.

18 Sanjeevi CB, Lybrand TP, Deweese C, et al. Polymorphic amino-acid variations in HLA-DQ are associated with systematic physical property changes and occurrence of Iddm. Diabetes 1995; 44: 125-131.
19 Kockum I, Sanjeevi CB, Eastman S, et al. Complex interaction between HLA DR and DQ in conferring risk for childhood type 1 diabetes. Eur J Immunogenet 1999; 26: 361-372.

20 Foley PJ, McGrath DS, Puscinska E, et al. Human leukocyte antigen-DRB1 position 11 residues are a common protective marker for sarcoidosis. Am J Respir Cell Mol Biol 2001; 25: 272-277.

21 Grunewald J, Brynedal B, Darlington P, et al. Different HLA-DRB1 allele distributions in distinct clinical subgroups of sarcoidosis patients. Respir Res 2010; 11: 25.

22 Rossman MD, Thompson B, Frederick M, et al. HLA-DRB1*1101: a significant risk factor for sarcoidosis in blacks and whites. Am J Hum Genet 2003; 73: 720-735.

23 Sato H, Woodhead FA, Ahmad T, et al. Sarcoidosis HLA class II genotyping distinguishes differences of clinical phenotype across ethnic groups. Hum Mol Genet 2010; 19: 4100-4111.

24 Hattori N, Niimi T, Sato S, et al. Cytotoxic T-lymphocyte antigen 4 gene polymorphisms in sarcoidosis patients. Sarcoidosis Vasc Diffuse Lung Dis 2005; 22: 27-32.

25 Spagnolo P, Renzoni EA, Wells AU, et al. C-C chemokine receptor 5 gene variants in relation to lung disease in sarcoidosis. Am J Respir Crit Care Med 2005; 172: 721-728.

26 Sato H, Williams HRT, Spagnolo P, et al. CARD15/NOD2 polymorphisms are associated with severe pulmonary sarcoidosis. Eur Respir J 2010; 35: 324-330.

27 Fischer A, Nothnagel M, Franke A, et al. Association of inflammatory bowel disease risk loci with sarcoidosis, and its acute and chronic subphenotypes. Eur Respir J 2011; 37: 610-616.

28 Grunewald J, Eklund A, Olerup O. Human leukocyte antigen class I alleles and the disease course in sarcoidosis patients. Am J Respir Crit Care Med 2004; 169: 696-702.

29 Ohara K, Okubo A, Sasaki H, et al. Intraocular manifestations of systemic sarcoidosis. Jpn J Ophthalmol 1992; 36: 452-457.

30 Evans M, Sharma O, LaBree L, et al. Differences in clinical findings between Caucasians and African Americans with biopsy-proven sarcoidosis. Ophthalmology 2007; 114: 325-333.

31 Baughman R, Costabel U, du Bois R. Treatment of sarcoidosis. Clin Chest Med 2008; 29: 533-548.

32 Pietinalho A, Ohmichi M, Hirasawa M, et al. Familial sarcoidosis in Finland and Hokkaido, Japan - a comparative study. Respir Med 1999; 93: 408-412. 